INPLASY

PROTOCOL

To cite: Yang et al. Moxibustion for lateral epicondylitis: a protocol of systematic review and meta-analysis of randomized clinical trials. Inplasy protocol 202150078. doi:

10.37766/inplasy2021.5.0078

Received: 19 May 2021

Published: 19 May 2021

Corresponding author:

Yang Zhijun

951350674@qq.com

Author Affiliation: Jiangxi University of Chinese Medicine

Support: Postgraduate Innovation Fund.

Review Stage at time of this submission: The review has not yet started.

Conflicts of interest: None declared.

\section{Moxibustion for lateral epicondylitis: a protocol of systematic review and meta-analysis of randomized clinical trials}

Yang, Z1; Yang, F2; Fu, W3.

Review question / Objective: To evaluate the efficacy and safety of moxibustion in the treatment of lateral epicondylitis. Eligibility criteria: Type of participants.: all participants included in the study were diagnosed with LE, regardless of age, sex, race, occupation, nationality. Type of interventions: moxibustion as a single intervention or major part of a combination therapy with other active intervention (eg, conventional medications, Chinese herbs, acupuncture and so on) will be included. There is no restriction on moxibustion method, acupoint selection. Type of comparators: the comparative interventions could be, no treatment, placebo or other active treatments (eg, corticosteroid injection, physiotherapy, anti-inflammatory drugs and so on). The retaining time, dosage and course of treatment are not limited. Types of outcome measures: the total effective rate, visual analogue scale (VAS), mayo elbow performance score (MEPS), grip strength, recurrence rate, adverse events.

INPLASY registration number: This protocol was registered with the International Platform of Registered Systematic Review and Meta-Analysis Protocols (INPLASY) on 19 May 2021 and was last updated on 19 May 2021 (registration number INPLASY202150078).

\section{INTRODUCTION}

Review question / Objective: To evaluate the efficacy and safety of moxibustion in the treatment of lateral epicondylitis.

Condition being studied: Lateral epicondylitis (LE), also known as tennis elbow, is a common musculotendinous degenerative disorder of the extensor origin at the lateral humeral epicondyle. Clinically, the typical manifestations include lateral elbow pain, pain with wrist extension, and weakened grip strength. To some extent, this condition severely affects people's quality of life and daily work. 
According to an epidemiological survey, the prevalence rate of LE is $1 \%$ to $3 \%$, and it can reach $7 \%$ in those people who repeatedly perform forearm rotation and vigorous wrist extension activities. Another survey has reported that $10.5 \%$ of manual workers may experience lateral elbow pain. Due to the high incidence, LE is gradually becoming a major public health issue. The pathogenesis of IE is controversial. In the past, most people thought that the pathological mechanism of LE was related to aseptic inflammation, but now it is considered to be tendon degeneration. In the pathological tissue of the extensor carpi radialis brevis (ECRB) tendon of patients with chronic LE, apoptotic cells and autophagy cells were found, and the number of those cells increase with the severity of lesion. Thus, it is currently believed that the basic underlying pathologic change is the presence of tendinosis tissue at the origin of ECRB. At present, the treatments of LE are divided into conservative and surgical treatment. Common conservative treatments include physical therapy, corticosteroid injection, platelet-rich plasma (PRP) injections and Non-steroidal anti-inflammatory drugs (NSAIDS). Physiotherapy is regarded as the first-line therapy for patients with LE, such as shock wave, laser, ultrasound and pulsed magnetic wave therapies. Nevertheless, there is no conclusive evidence that these physical therapies can have a beneficial effect on LE. Corticosteroid injection and NSAIDS can greatly relieve pain within a few weeks. However, there will be adverse effects occur in the long term. Surgical approaches of LE include open, arthroscopic and percutaneous. On the one hand, surgery will add new trauma to patients. On the other hand, the extra cost of surgery will bring financial burden to family. Therefore, more and more LE patients pay attention to some alternative therapies, such as moxibustion and acupuncture. Moxibustion, as a characteristic Chinese medicine therapy, has been developed for thousands of years. Modern research shows that it can relieve pain, anti-inflammatory, enhance immunity and improve blood circulation.
Nowadays, plenty of clinical studies have reported that moxibustion has a good effect in the treatment of LE.

\section{METHODS}

Search strategy: Eight relevant electronic databases include PubMed, Embase, Web of Science, the Cochrane library, CNKI, SinoMed, VIP and WanFang databases from their inception until May 2021 will be searched. The language within in Chinese and English. We will strictly follow the PRISMA statement, the following search terms will be used: moxibustion, lateral epicondylitis, tennis elbow, lateral elbow pain, etc. The retrieval strategy will be adjusted according to different databases. To avoid omissions, we will manually search for other potential articles in the reference list of included studies. Besides, We will search for eligible trial which is ongoing or unpublished.

Participant or population: All participants included in the study were diagnosed with LE, regardless of age, sex, race, occupation, nationality.

Intervention: Moxibustion as a single intervention or major part of a combination therapy with other active intervention (eg, conventional medications, Chinese herbs, acupuncture and so on) will be included. There is no restriction on moxibustion method, acupoint selection.

Comparator: The comparative interventions could be, no treatment, placebo or other active treatments (eg, corticosteroid injection, physiotherapy, anti-inflammatory drugs and so on). The retaining time, dosage and course of treatment are not limited.

Study designs to be included: All relevant randomized clinical trials (RCTs) published in English and Chinese about moxibustion for LE can be included, without restriction of blinding and publication type.

Eligibility criteria: Type of participants.: all participants included in the study were diagnosed with LE, regardless of age, sex, 
race, occupation, nationality. Type of interventions: moxibustion as a single intervention or major part of a combination therapy with other active intervention (eg, conventional medications, Chinese herbs, acupuncture and so on) will be included. There is no restriction on moxibustion method, acupoint selection. Type of comparators: the comparative interventions could be, no treatment, placebo or other active treatments (eg, corticosteroid injection, physiotherapy, anti-inflammatory drugs and so on). The retaining time, dosage and course of treatment are not limited. Types of outcome measures: the total effective rate, visual analogue scale (VAS), mayo elbow performance score (MEPS), grip strength, recurrence rate, adverse events.

Information sources: PubMed, Embase, Web of Science, the Cochrane library, CNKI, SinoMed, VIP and WanFang databases.

Main outcome(s): The total effective rate, visual analogue scale (VAS).

Additional outcome(s): Mayo elbow performance score (MEPS), grip strength, recurrence rate and adverse events.

Data management: All literature retrieved from electronic databases will be managed by NoteExpress 3.2.0 software, and the duplicated literature will be excluded. According to pre-designed inclusion and exclusion criteria, two reviewers (YZJ and FWQ) discard unqualified articles by browsing the title and abstract. Then, they download the full text of all possible related studies for further inspection to make a final decision. If the information contained in the article is not sufficient to determine eligibility, we will try to contact the author of the original report for more detailed information. In this process, differences will be resolved through discussions or consensus with the third reviewer.

Quality assessment / Risk of bias analysis: Two reviewers will independently assess the methodological quality of the included studies based on the bias risk assessment tool in the Cochrane Collaborative Network System Reviewer's Systematic Evaluation Intervention Manual. Researchers will evaluate 7 items, including random sequence generation, allocation concealment, blinding of participants, personnel blinding of outcome assessment, incomplete outcome data, selective outcome reporting and other sources of bias. The assessment of each aspect will be classified into 3 levels, including high risk, low risk and unclear risk. In this process, the disagreement will be resolved through communication with the third reviewer.

Strategy of data synthesis: We will use RevMan 5.4 software for statistical analysis. The result will be presented as risk ratio with a $95 \%$ confidence interval (CI) for dichotomous data and weight mean difference (WMD) or standard mean difference (SMD) with a $95 \% \mathrm{Cl}$ for continuous data. When meta-analysis is not possible, we will perform descriptive analyses. Besides, we will choose the 12 test to evaluate the heterogeneity of the included studies. When $1250 \%$, indicating lower heterogeneity, and random-effects model will be selected. If $12>75 \%$, we will choose subgroup analysis or sensitivity analysis to look for possible sources from clinical and methodological aspects.

Subgroup analysis: If necessary, we will conduct subgroup analyses based on age, gender, duration of treatment, follow-up time, etc.

Sensitivity analysis: If there is a high degree of heterogeneity ( $12>75 \%$ ) in our study, we will perform sensitivity analysis based on sample size, methodological quality and missing data results. Further, the analysis will be repeated after excluding studies with poor methodological quality.

Language: English and Chinese.

Country(ies) involved: China.

Keywords: Lateral epicondylitis, moxibustion. 
Dissemination plans: The results of this study will be disseminated through peerreviewed publications and presented at relevant meetings.

Contributions of each author:

Author 1 - Yang Zhijun - The author drafted and improved the manuscript.

Email: 951350674@qq.com

Author 2 - Yang Fengyun - Revise this protocol; search strategy.

Email: yang_20190116@163.com

Author 3 - Fu wenqiang - Data collection; analysis of results. 